\title{
Etat bureaucratique, pouvoirs locaux et développement en Afrique
}

\author{
Par Jean Njoya, Yaoundé
}

Le type idéal bureaucratique est conçu comme un schéma d'organisation rationnelle centré sur les relations formelles qui commande le fonctionnement d'une Administration pour en assurer l'efficacité ${ }^{1}$. Ce schéma selon Max Weber surpasse toutes les autres formes d'organisations administratives en prévision, en rapidité, en rigueur et en efficacité ${ }^{2}$.

C'est un modèle qui suppose une unité structurelle de l'Etat et une subordination hiérarchique reposant sur un système de règles abstraites, impersonnelles et objectives conformes à l'idée que la bureaucratie est un ensemble de relations entre fonctions et rôles et non entre individus ${ }^{3}$.

Cette conception unitaire du pouvoir par son inspiration jacobine suppose que l'autorité centrale se pose en un seul centre d'impulsion politique ${ }^{4}$. Le paradigme centre-périphérie s'appréhenderait dès lors comme un processus d'imposition unilatérale du centre à la périphérie. R.DRAÏ utilise le concept d'«administration séparée» pour traduire le caractère unidimensionnel des rapports entre pouvoir central et la périphérie; l'administration centrale serait la projection institutionnelle du mythe Kafkaïen : le «château» constituant le centre cosmique et «sacral» par rapport auquel s'ordonnent toutes les volontés sans aucune possibilité de dialogue avec son environnement ${ }^{5}$.

J. Chevalier / D. Loschack, Science administrative, Paris, LGDJ, T II, 1978, p. 11.

2 M. Weber, Rhizome et Société, Paris, Plon, p. 223-228.

R.K. Merton, «Bureaucracy, structure and personality » in reader and Bureaucracy, the free press of Glen coe. III, 1940.

4 G. Burdeau, Traité de Science politique, Paris, LDGJ, 1968, III, p 571.

5

R. Draï, «Science administrative et relations humaines » Bull. IIAP N³6. Oct. - Déc. 1975, p 7. 
Cette vision s'inscrit du reste dans une vieille tradition intellectuelle développementaliste qui appréhende la construction de l'Etat dans une perspective institutionnelle, indépendamment de sa socialisation ${ }^{6}$.

Le programme d'ajustement structurel avec ses conditionnalités draconiennes, la mondialisation avec ses logiques monétaires ont entraîné une profonde mutation de l'Etat dans le sens d'une redéfinition de son rôle dans les tissus économique, social et culturel. Ces logiques structurent le rapport entre le pouvoir central et son environnement, rapport désormais perturber par le foisonnement des acteurs concurrentiels, et marqué par une forte hétérogénéité.

La littérature scientifique a accordé une attention distraite au monde rural et a consacré bien de ses analyses aux mutations politiques ${ }^{7}$ comme si le champ politique opérait en marge du champ social. Seul Goran Hyden a initié une étude pionnière de facture marxiste sur la société tanzanienne des années 70 . Il posait l'hypothèse polémique selon laquelle les paysans africains ne seraient soumis ni par la pénétration du capitalisme, ni par des régimes socialistes : cette insoumission serait selon Hyden un obstacle au développement : c'est ce qu'il a appelé «un captured peasantry» (la paysannerie non capturée). Le paysan aurait ainsi opéré un «choix d'évasion » (exit option) par rapport aux politiques définies par le pouvoir central $^{8}$. Le concept «d'économie d'affection» qu'il forge expliquerait cet enfermement autarcique de la paysannerie africaine. Soutenir l'hypothèse d'un isolationnisme du paysannat est une approche singulièrement appauvrie. Visiblement, elle a partie liée avec le phalanstère de Charles Fourier ${ }^{9}$ qui prônait l'avènement des isolats communautaires paradisiaques. La carence analytique est donc notable. Le modeste essai que nous proposons ici a valeur suggestive, et ambitionne d'introduire commodément le lecteur à l'un des phéno-

Voir Mahmood Madani, citizen and subject, contemporary Africa and the legacy of late colonialism. Princeton University Press, 1996. voir également James Scott, seeing like a state, Yale University press 1998.

Voir à ce sujet Célestin Monga, L'émergence de nouveaux modes de production démocratique en Afrique Noire. Afrique 2000. Revue Africaine de politique internationale. $\mathrm{N}^{\circ} 7$; pp 111-125. voir également S .A. A Lao élections et systèmes électoraux dans le processus de démocratisation en Afrique. Colloque European African relations in the 1990e s, Munich, 24-27 mai.

Goran Hyden Beyond Ujamaa in Tanzania ; undevelopment and uncaptured peasantry, Londres, Heinemann, 1980, p 21 et 121. voir également G. Hyden, The resilience of the peasant mode of production, the case of Tanzania, in R.H. Bates / M.F. Lofchie (eds), Agricultural development in Africa: issues of public policy, New York, Praeger, 1980, pp. 218-243.

Charles Fourier était un communiste français; le système de Fourier ou Fouriérisme, prévoit l'association des individus dans des phalanstères, groupes humains harmonieusement composés en vue de procurer à chacun de leur membre le bien-être par le travail librement consenti. 
mènes sociaux sécrétés par la libéralisation politique et économique : l'irruption des organisations paysannes.

La périphérie est primordialement animée par la paysannerie qui aujourd'hui se constitue progressivement en forces sociales; elle est le catalyseur des aspirations latentes du monde rural. En science politique, les organisations paysannes se rattachent au phénomène des groupes de pression conçus comme des structures d'agrégation et d'articulation des intérêts ${ }^{10}$. L'étude de ces associations infère une analyse de leur condition d'émergence et une appréciation de leur capacité d'action sur les pouvoirs publics (I)

Mais, cette approche reste descriptive si on ne conçoit pas ces organisations comme des acteurs en réseaux ${ }^{11}$ impliqués dans un champ interactionniste qui structure le «pouvoir périphérique » et modifie sa relation unilatérale avec le centre.

L'hypothèse est donc que sous l'action de ces groupes de pression (dont les O.P.), la relation centre-périphérie subit une mutation et passe insensiblement du modèle unilatérale au modèle échangiste ${ }^{12}$. On conçoit donc que cette relation puisse être cernée à travers un dynamique des rapports bijectifs (II).

\section{Les organisations paysannes comme catégories rebelles au modèle bureautique d'intervention étatique}

Les organisations paysannes sont des «groupes d'intérêts » qui se muent insensiblement en «groupes de pression» lorsque le pouvoir politique tente d'annihiler leur action en les enfermant dans un carcan de normes juridiques ; 1'expression «groupe de pression » vient de la science politique américaine et caractérise l'action et la place des «groupes d'intérêts » dans la structure de pouvoir ${ }^{13}$.

C'est l'approche institutionnelle qui a dans une certaine mesure biaisée l'analyse sociologique du phénomène des groupes sociaux. Or celle-ci aurait gagné à s’inscrire dans le sillage de la sociologie dynamique et appréhender le réel en s'intéressant au «processus » plutôt 
qu'aux structures, aux groupes qui contrôlent l'exercice du pouvoir plutôt qu'à l'aménagement constitutionnel de celui-ci ${ }^{14}$.

L'enfermement juridique de l'expression des groupes crée une distorsion qui limite l'intensité des échanges entre le pouvoir central et son environnement social en multipliant les obstacles et les courts circuits. Cette coupure avec l'environnement était encouragée par l'idéologie de l'intérêt général qui voit dans la neutralité et l'indépendance de l'Administration, la condition essentielle de son efficacité.

Aussi, le foisonnement des organisations paysannes déteint-il considérablement sur la dynamique de l'Etat bureaucratique, et corrélativement sur les nouvelles régulations sociales en Afrique ; elles imposent une reformulation du modèle centre-périphérie.

Une analyse des conditions de leur émergence (A) nous permettra de cerner les logiques constitutives de leur assise locale (B).

\section{A. Contexte et facteurs de l'émergence subversive des organisations paysannes : l'environnement économique comme facteur structurant de la périphérie rurale}

L'émergence des organisations paysannes est largement tributaire du contexte général de la libération politique et économique. Elle résulte d'une combinaison des déterminants internes et internationaux qui amputent l'Etat d'un pan entier de son domaine de déploiement. C'est dire qu'elles tendent à produire un marché mondial unique pour les biens, les services, le capital et le travail ${ }^{15}$ et au désengagement de l'Etat. Ces logiques leur imposent une dynamique qui les soustrait de l'emprise étatique. Ce mode populaire de défit et de substitution révèle les infortunes d'un «rhizome rhizome » idéologiquement enclin à la pénétration juridique totale de la périphérie.

L'Analyse Institutionnelle est jugée trop juridique, trop formelle, et beaucoup trop optimiste. Dans le domaine des «processus politiques ». il faut donner vogue à Bentley qui en 1908 rattachait l'étude des groupes aux «processus décisionnels ( $F$. Bentley, The process of government, a study of social processures, Chicago, 1908).

S. Berger, «Globalisation and politics », Annual political science Review, 3, 2000, 44, p.43-62. 


\section{Les logiques de la mondialisation et la « rébellion sociale » des organisations paysannes}

Soucieux de rendre compte de l'existence d'une «société mondiale», John Burton propose le modèle de la «toile d'araignée» ${ }^{16}$ pour préciser que le débat sur les acteurs des relations internationales s'est déplacé du problème de l'énumération des acteurs vers ce que les constructivistes appellent le débat «agent structure » ${ }^{17}$. Toiles tissées d'innombrables flux commerciaux, de mouvements des populations, d'échanges culturels, des relations entre ville ou village, des interactions sociales entre communautés.

Les organisations paysannes s'impliquent ainsi dans l'immédiateté d'une dynamique mondiale qui commande une investigation depuis l'intérieur au sein de l'acteur transnational. Le monde rural subit directement ou indirectement les dégâts collatéraux de son insertion dans une économie ultra-libérale. La mondialisation lui impose une configuration largement tributaire des logiques commerciales, productives et financières ${ }^{18}$.

En effet, l'économie mondiale est passée sensiblement d'une problématique centrée sur les dotations naturelles (agricoles, minières) et factorielles (travail, capital) à une problématique focalisée sur les structures et l'efficacité productives. Ainsi est privilégiée la qualité des spécialisations internationales appréciées non plus seulement par rapport aux conditions d'offre, mais aussi par rapport à leur adaptation à la demande mondiale. Et, longtemps masquée par les approches en termes de divisions internationales du travail, le principe concurrentiel donne prise à l'analyse des déterminants de la compétitivité. Ces logiques commerciales font poindre à l'horizon le spectre d'un monde paradisiaque ou d'un «marché de tout l'univers » selon l'expression de SISMONDI, débarrassé de tout obstacle aux échanges.

De la même manière, les logiques productives se traduisent par la concentration des investissements au sein de la triade ${ }^{19}$, avec une prépondérance des investissements directs croisés. Dans la même veine, les logiques financières impliquent une mobilité du capital sous

J. Burton, Systems, states, diplomacy and rules, Cambridge, Cambridge university pres, 1968.

17

Le débat agent-structure est une question classique en philosophie. Il est relatif aux rapports entre agent et structure dans son expression la plus simple. Qui de l'acteur ou de la structure détermine qui ? structure, constructivistes privilégient les deux dimensions en posant l'hypothèse de la constitution et de la codétermination de l'agent par la structure et vice-versa, J. Adda, La mondialisation de l'économie, T2. Problème et perspectives Ed. La découverte. Paris, 1996. pp. 6 et 55.

M.E. Touna, «Qu'est-ce que la mondialisation ? » in La mondialisation de l'éconmoie camerounaise, Yaoundé, Saagraph et Friedrich Ebert, 1998, p. 23.

19

La triade est un groupe de 3 pôles composés des Etats Nord Américains, de l'Europe de l'Ouest et du Japon. 
sa forme monétaire et une libéralisation complète des mouvements des capitaux dont la principale conséquence est l'émergence des eurodollars.

Dans ce contexte, la part des économies des pays en développement comme zones d'accueil des investissements internationaux est faible et déclinante ${ }^{20}$. Grosso modo, l'internationalisation croissante des économies au cours de ces dernières années s'est manifestée par une aggravation de la précarité des populations africaines ${ }^{21}$.

Les organisations paysannes nées dans ces conditions doivent être appréhendées dans le sens que leur confère Bertrand Badie ; c'est-à-dire comme des «communautés de responsabilité » qui traduisent la volonté de la paysannerie de construire une communauté dénouée de ses allégeances nationales, pour apparaître comme porteuse d'une culture propre, au particularisme persuasif ${ }^{22}$. Le monde rural se transforme ainsi en un espace de recomposition et de chevauchement transcendant les frontières et sécrétant des pouvoirs nouveaux. Certains auteurs projettent même une réconciliation du mondial et du local et proposent de substituer au vocable «mondialisation », le mot métaphoriquement expressif de «Globalisation » ${ }^{23}$, plus apte à expliciter l'inextricable liaison entre le mondial et le local. La mondialisation se trouve ainsi structurée par la dynamique des productions locales, en même temps qu'elle s'équilibre dans la formation des régions transversales.

Même si les organisations paysannes en Afrique n'ont pas encore l'envergure de la Fédération nationale des syndicats d'exploitants agricoles en France ou de la puissante rhizome rhizome verband en Allemagne, elles tendent déjà à contrevenir à la souveraineté de l'Etat en desserrant les contraintes hiérarchiques qui annihilent les capacités d'innovation rurale. Ainsi parle-t-on d'une «bifurcation » du système international impliquant la coexistence d'un «système stato-centré et d'un système multi-centré puissant, mais plus décentralisé » 24 .

Il faut souligner que 1/3 du stock mondial était localisé dans ces pays en 1960, 1/4 en 1980, 1/5 en 1990 donc la moitié en Amérique Latine et 1/3 en Asie. Par rapport à l'avant guerre, le déclin est plus frappant puisque les 2/3 environ du stock mondial étaient localisés en 1914 comme en 1938 dans les régions dites en développement (M. Touna, op. cit).

21 Voir les indicateurs du développement humain de 1991. 1994, 1997 du PNUD.

22 B. Badie, Un monde sans souveraineté, Les Etats entre ruse et responsabilité, Paris, Fayard. 1999. p. $168-169$.

$R$. Robertson, «Globalisation time space and homogeneity-heterogeneity, in S. Theatrestone / $R$. Robertson (éds.) Global modernities, Londres, Sage, 1995, p. 1-24.

James N. Roseau, Along the Domestic - foreign frontier: Exploring governance in a turbulent world, Cambridge University press, 1997, p.11. 
Dans cette frénésie libérale, les organisations paysannes se posent comme de véritables structures de recomposition de la périphérie rurale. Et bien plus selon la banque mondiale, il est difficile, voir impossible de faire reculer la pauvreté sans une croissance rapide du secteur agricole $^{25}$.

Si les logiques de la mondialisation ont modulé une «échancrure » vers l'inscription dans le corps de l'Etat des acteurs concurrentiels, ce dernier (l'Etat) est resté conforté dans l'illusion tenace d'une maîtrise totale de la périphérie.

\section{Les prétentions d'une pénétration totale de la périphérie : L'illusion d'une mise en ordre de l'espace rural par le centre}

L'illusion qu'il n'y a au sein de l'Etat qu'un «seul centre d'impulsion politique, économique et culturel» est tenace ; elle donne du corps à l'idée que la construction de structure suppose à l'avenant l'imposition de sa volonté unique au corps social. C'est un modèle de gestion wébérien qui privilégie la dimension unilatérale de la relation centre-périphérie. C'est le modèle de société bloquée dont le fonctionnement monocratique fait douter de l'existence d'une sphère locale distincte ${ }^{26}$. Cette façon de concevoir l'Etat est d'essence jacobine et implique qu'il suffit d'instaurer des relais périphériques pour que la volonté étatique s'impose avec une belle facilité.

Dans les structure Africains préoccupés aux premières heures de l'indépendance par les impératifs du développement économique, de l'intégration et de l'unité Nationale, la tutelle exercée sur les relais locaux et les établissement publics ont rendu leur autonomie plus nominale que réelle; même les entreprises publiques considérées comme un instrument essentiel de son action sur les structures économiques, étaient soumises à de lourdes suggestions qui obéissaient à la logique d'une économie de commandement. Il est donné à noter au demeurant qu'il existait une consubstantialité entre l'idéologie de la construction nationale et les institutions politiques de la plupart des structure africains ${ }^{27}$. Cette obsession unitariste transformait le discours sur l'unité nationale en une sorte d'énoncé «constatif», le « dire » se muant insensiblement en « faire ${ }^{28}$.

J. Hayward «Société bloquée et collectivités locales en GB. La reforme rétrograde de 1972 », Bull. de 1'I .A.P, Juillet-Septembre 1976, n 39. p. 41. Institutions politiques de l'Etat camerounais », in Gerard Conac, Dynamiques et finalités des droits africains. Colloque Sorbonne, Paris,1980, pp. 442-466.

J.L. Austin, Quand dire c'est faire, Paris, Seuil, 1970. 
Par inférence, le monde rural s'est trouvé ainsi moulé dans une sorte «de lit de Procuste » idéologique. L'une des manifestations de cette emprise étant l'option étatique pour un développement rural par projet. Ce concept aux acceptions ambivalentes a justifié le drainage de l'essentiel des ressources destinées au secteur rural depuis l'indépendance ${ }^{29}$.

En Afrique francophone, le caractère centralisé de la relation centre-périphérie entre le pouvoir central et le monde paysan est un héritage colonial. Les organisations paysannes en tirent d'ailleurs leur origine. On peut trouver une préfiguration dans les sociétés indigènes de prévoyance (SIP) créée en 1910 au Sénégal, à partir des modèles expérimentés en Algérie et en Tunisie ${ }^{30}$. Ces sociétés ont reçu des appellations diverses justifiées par les disettes sporadiques. C'est dans la décennie 70 qu'on a vu se développer sous des dénominations variées de véritables organisations paysannes.

Dans l'ensemble, la naissance du mouvement paysan africain de la période post-coloniale, s'est opérée en marge du modèle du groupe des «équitables pionniers de Rochdale » qui, en 1844 dans le contexte de l'Angleterre industrielle instituait et amendaient eux-mêmes leur organisation ${ }^{31}$.

Au Cameroun, la politique appliquée au monde rural participait d'une stratégie de «pénétration politique » de la périphérie d'inspiration développementaliste ${ }^{32}$. Le développement par projet était ainsi considéré comme un lieu de construction de structure avec ses trois aspects : le volet idéologique, l'intégration économique et sociale et la dimension distributrice entre les segments de l'élite. L'Etat construisait la nation par ce biais en tenant de fédérer les divers segments de l'élite par la participation géoethnique à l'exercice du pouvoir $^{33}$. Telle a été la spécificité de l'approche camerounaise du développement rural. Un développement qui mobilise les symboles, les mythes et l'histoire : mythe de la technocratie porteuse du progrès général et réalité d'une décolonisation dénouée par une guerre civile maîtrisée par le colonisateur et d'une unification fondatrice entre anglophones et francophones ${ }^{34}$, mythe de la diversité des potentialités valorisables, mythe de la spécificité camerounaise en Afrique. Le développement procède donc de l'Etat qui exerce sa capacité

A. Marlty, «Les organisations coopératives en milieu pastorale », In cahier des sciences humaines - Vol 26 - N¹-2. 1990, p. 122.

31 Marlty, op cit, p. 124.

$S$. Ndembou, «Le développement rural par projet à l'épreuve du désengagement de l'Etat », in Courade Georges, Le village camerounais à l'heure de l'ajustement, Paris, Karthala, 1994, p.295. 
régulatrice sur l'ensemble du monde rural ; c'est la loi de 1968 sur les sociétés de développement qui fait entrer tous les projets déviants à leur origine, dans l'ordre bureaucratique où tout procède du pouvoir central; puis s'en est suivie une frénésie décrétale donnant cours à une multiplicité de projets dans le monde rural : décret $\mathrm{N}^{\circ} 71 / \mathrm{DF} / 74$ portant création de la SEMRY, décret $\mathrm{N}^{\circ} 70 / \mathrm{DF} / 529$ portant création de l'UNVDA, décret N73/694 (création de la SODEBLE), décret $\mathrm{N}^{\circ} 73 / 235$ portant création de la SODERIM etc.).

A la même période est nationalisée le rhizome development corporation (CDC), l'un des plus gros employeurs dans le secteur agro-industriel.

Sur le plan structurel, on s'aperçoit que cette modalité d'organisation du monde rural est conforme au modèle wébérien de bureaucratie. Toutes les sociétés de développement ainsi créées étaient placées sous la tutelle du Ministère du plan ou du Ministère de l'agriculture. Bien que dotée d'organes de consultation, la relation centre-périphérie demeurait verticale et ne donnait lieu aucun échange bijectif. Or, l'une des principales figures du rapport entre l'Etat et la société, est sans doute, la coproduction de l'ordre politique, culturel et économique par les deux secteurs ${ }^{35}$.

Bien plus, l'Etat pouvait aussi revendiquer avec succès le monopole de la contrainte par sa «capacité d'extraction» ${ }^{36}$ grâce au prélèvement effectué sur le secteur agro-exportateur aux prix rémunérateurs. Cette taxation était d'ailleurs jugée trop lourde par la Banque mondiale $^{37}$ et opérait tantôt de façon explicite (fixation des prix à la production, taxation des exportations et des intrants), tantôt de façon implicite (taux de change surévalués, baisse du prix à l'exportation).

Les sociétés de développement ont même fonctionné comme des puissantes machines bureaucratiques s'inspirant de la distinction manichéenne d'essence taylorienne entre la fonction de conception et la fonction d'exécution. En effet, dans la gestion de ces sociétés, trois niveaux de responsabilité étaient repérables : la conception au niveau des ministères, la supervision de l'application des décisions du niveau de la direction générale et de l'exécution au niveau des encadrés. La rigidité d'un tel ordonnancement hiérarchique exclut d'emblée la dimension participative de la gestion à cause d'une rationalité toute monolithique qui présidait au fonctionnement des sociétés de développement. Bien plus, en raison d'un effectif pléthorique composé des fonctionnaires et agents de l'Etat, celles-ci apparaissaient comme une projection de la Fonction Publique dans le monde rural. Les

L. Structure, L'Etat ailleurs, op.cit, p.22. 
sociétés de développement étaient coiffées par un conseil d'administration au sein duquel les représentants des «encadrés » «faisaient de la figuration symbolique » ${ }^{38}$; la direction générale de l'entreprise s'apparentait quant à elle à une mini-administration centrale composée essentiellement d'agents et de fonctionnaires de l'Etat, souvent fort nombreux occupant les postes clés. Ainsi, la société de développement passe-elle insensiblement d'une «administration de mission» à une «administration de gestion» 39 .

Jean Coussy observe que la politique économique du Cameroun repose sur la pratique de la gestion néo-patrimoniale avec une affectation des financements pour des fins de consommation des gestionnaires et de leurs mandats ${ }^{40}$. C'est une économie de rente limitée à quelques fonctionnaires et agents de l'Etat ayant développé une technique d'affectation des financements pour des fins de consommation des gestionnaires et de leurs mandats; une économie où les fonctionnaires et agents de l'Etat ont une certaine fascination pour ces grandes sociétés, et où la part allouée aux crédits de fonctionnement était nettement plus importante que celle allouée aux crédits d'investissement. Les grilles de salaire étaient par conséquent plus élevées que celle de la fonction publique ${ }^{41}$.

Cette «économie de rapines» résultait également de l'irresponsabilité de l'Etat et du concessionnaire. En effet, toutes les sociétés de développement se voyaient concéder par le cahier des charges le droit de percevoir des redevances leur permettant le remboursement des emprunts contractés; de même qu'était prévu l'apport par l'Etat d'un fond de réserve et d'une subvention pour la couverture des charges d'entretien et d'exploitation. Le bilan est largement négatif comme l'indique le rapport sur le coût financier de maintenance des sociétés de développement rural (tableau ci-dessous). L'Etat n'ayant honoré qu'en partie ses obligations. La SEMRI par exemple n'a perçu, au titre de cette mission que des rares subventions d'exploitation provenant de la caisse de stabilisation sans la moindre contribution au fonds de réserve.

S. Ndembou, « Développement rural par projet à l'épreuve du désengagement de l'Etat », Op.cit p. 297.

E. Pisani, «Administration de mission et Administration de gestion », RFSP. 1954.

40

J. Courssy, «Le conservatisme de la politique économique du Cameroun après l'indépendance : Origine, rationalités et conséquence » in P. Geschiere, L'économie politique du Cameroun, Perspective historiques, Leiden. African studies center, 1-4, Juin 1988.

Voir N.R. Tsafack, « Mondialisation et détermination des salaires dans le secteur public » in Touna Mama, La mondialisation et l'économie camerounaise, F. Ebert 1998 pp. 285-310. En effet, à niveau égal, un cadre d'une société parapublique touchait deux à trois fois plus que son homologue de la fonction publique sans compter les avantages en nature supportés par l'entreprise. 
Coût financier de la maintenance de quelques sociétés de développement rural (en millions de francs CFA 1988-1989)

\begin{tabular}{|l|c|c|c|}
\hline Nom & Dépenses & Revenus & Déficits \\
\hline CDC & 2.390 & 1.540 & 850 \\
\hline HEVECAM & 4.100 & 3.400 & 700 \\
\hline MIDENO & 1.480 & 0 & 1.480 \\
\hline MIDEVIV & 4.300 & 370 & 3.930 \\
\hline MIDO & 500 & 0 & 500 \\
\hline SEMRY & 11.430 & 2.830 & 8.600 \\
\hline SODECAO & 12.500 & 2.400 & 10.100 \\
\hline SODECOTON & 41.000 & 27.280 & 13.720 \\
\hline SODENKAM & 930 & 160 & 770 \\
\hline SODERIM & 1.070 & 90 & 980 \\
\hline UNVDA & 4.500 & 3.900 & 600 \\
\hline WADA & 1.010 & 690 & 320 \\
\hline ZAPI-EST & 3.600 & 2.800 & 800 \\
\hline TOTAL & $\mathbf{8 8 . 8 1 0}$ & $\mathbf{4 5 . 4 6 0}$ & $\mathbf{4 3 . 3 5 0}$ \\
\hline SOUזC:CONi & & & \\
\hline
\end{tabular}

Source : Commission de réhabilitation des sociétés.

Dans ce contexte, toute initiative rurale rentrait dans l'ordre bureaucratique en ne laissant aucun interstice d'autonomie aux paysans. La rébellion du rural dans la conjoncture actuelle de libéralisation, montre que l'ordre économique, politique et culturel est moins une imposition du centre à la périphérique qu'une coproduction de l'Etat et de la société.

\section{B. La construction étagée d'un pouvoir paysan local}

L'encadrement du monde paysan va ainsi passer d'une continuité marquée par l'emprise juridique de l'Etat à une anarchie pathologique traduisant l'état d'anomie d'une conception unilatérale de la relation centre-périphérie.

Même si la centralité d'un pouvoir est une nécessité et une donnée caractéristique de notre époque, les structure nouveaux tendent plus ou moins aujourd'hui à traduire les éléments de modernité dans le «langage propre de la culture réceptrice » ${ }^{42}$; c'est-à-dire les logiques sociales propres de la périphérie. Ceci est d'ailleurs conforme aux exigences actuelles des politiques publiques 
La construction d'un pouvoir paysan s'inscrit dans les scénarios de l'ajustement structurel qui touche à trois dimensions essentielles : le P.A.S comme fait social où s'imbriquent le politique, le social et l'économie. Il suppose la mise en place d'une méthodologie articulant les dimensions micro avec les dimensions macro ${ }^{43}$. Quatre points essentiels touchant directement le monde rural sont retenus pour une relance de la croissance dans le cadre de l'économie de marché.

- La privatisation progressive des activités de développement agricole.

- La responsabilisation accrue des agriculteurs et la création des exploitations agricoles de moyenne importance supposées être plus compétitives que les exploitations familiales.

- La protection de la production agricole

- La protection de la production nationale.

Dans cette nouvelle vulgate néo-coloniale, il y a comme une transformation de l'Etat en «simple agent du processus de la mondialisation» ${ }^{44}$. De la logique de l'Etat-providence Keynésien, il est embarqué dans la spirale des contraintes de l'Ajustement Structurel. L'Etat devient un législateur offrant aux nouveaux promoteurs du monde rural un espace de liberté d'action. L'émergence d'un pouvoir paysan est la résultante de l'éclatement des monopoles qui pesaient lourd sur le monde villageois.

Cette déconstruction de l'emprise juridique de l'Etat qui ouvre les vannes au foisonnement des organisations paysannes s'analysent aujourd'hui comme un «mode populaire» de défi ${ }^{45}$ dont la dynamique parfois anarchique hypothèque leur efficacité.

L'émergence des Organisations paysannes bien que problématique s'inscrit dans ce que Bertrand Badie appelle les «communautés de responsabilité » dont la survenance tient à l'irruption d'enjeux nouveaux et des problèmes inédits ${ }^{46}$.

1. La construction étagée et problématique du pouvoir paysan

A. Kalette, «Pour un programme pluridisciplinaire sur les politiques d'ajustement en Afrique de l'Ouest », Chronique du Sud, N 4 Janvier 1991., Paris, Orstom, pp. 23-32. « actions politiques des sans importance qui résistent aux institutions, s'en détournent, les détournent et s'y opposent » 'l'Etat au Cameroun, Paris PFSNP, 1985, p 256.

B. Badier, Un monde sans souverainété, op cit, p. 165. 
Georges Burdeau considère les «groupes d'intérêt » comme de forces politiques organisées $^{47}$. Sans doute ne le deviennent-elles que lorsque la tactique suivie par le groupe pour obtenir la satisfaction des intérêts qu'il sert le conduit à s'engager dans la voie politique.

C'est une perspective nouvelle liée aux mutations qui dérivent de l'évolution même des communautés humaines. L'influence directe ou détournée que les O.P exercent sur l'action gouvernementale et sur l'opinion publique ${ }^{48}$ leur fait perdre incontestablement leur nature prioritairement locale. Elles se démultiplient, se complexifient, se superposent, se chevauchent parfois et aspirent au-delà de leur marquage identitaire et de la défense de leurs intérêts, à faire de la politique autrement. Comme «force politique organisée », le pouvoir paysan se nourrit de la dynamique des productions locales.

Mais, la naissance et la consolidation progressive de ce pouvoir se sont opérées par paliers. De glissement en glissement, les O.P sont passées de la structure atomique à des polarisations fédératives. Par ce jeu d'ondes, elles mènent aujourd'hui à l'échelle mondiale une insidieuse révolution tranquille.

\section{a. La construction microscopique du pouvoir paysan}

L'expression « Organisation paysanne » renvoie à des structures gérées au moins en théorie par des paysans et dénommées fort diversement : coopératives, associations, groupements, comités, unités, foyers, amicales etc... ${ }^{49}$; cette définition insinue non seulement le caractère fortement diversifié des O.P, mais aussi leur émergence quasi-anarchique impulsée au Cameroun par les lois du 19 décembre 1990 ; lois ayant déverrouillé le dispositif dirigiste de l'Etat. Le monde rural s'est trouvé atomisé par une extrême variété d'organisations ; l'Etat s'étant longuement investi de manière très discontinue dans le monde paysan, déployant les efforts dans les zones où il pouvait espérer tirer de l'agriculture des revenus substantiels à partir des mécanismes plus ou moins complexes basés sur des systèmes de catégorie « groupe de pression » est bien utile étant donné son élasticité.

A. Mathiot, Les «Pressures Groups » aux EU ?, Révue Française de Science Politique, 1982, p. 430. en effet, les groupes de pression ne sont rien de plus que d'innombrables groupements, associations, syndicats ou sociétés qui défendent les intérêts communs à leurs membres, s'efforcent par tous les moyens en leur pouvoir, directs ou détournés d'influencer l'action gouvernementale et législative, d'orienter aussi l'opinion publique.

A. Marty, «Les organisations coopératives en milieu pastoral : héritage et enjeux », in Cahier des sciences humaines 26 (1-2) 1990, p. 121. 
prélèvement $^{50}$. Cette pénétration sélective de l'Etat en brousse doublée d'un environnement économique particulièrement défavorable a engendré des « ripostes paysannes à la crise ${ }^{51}$.

Mais, comment cerner cette réalité sociologique extrêmement foisonnant, microscopique et complexe ? Autrement dit, quelles sont les catégories d'O.P qui peuplent aujourd'hui l'espace rural, le recomposent et qui organisent une véritable rébellion contre les canons de la souveraineté stato-nationale?

Evidamment, toute classification est arbitraire ; mais elle l'est moins lorsque la construction des catégories aboutit à une représentation formelle et cohérente, rendant compte des traits caractéristiques. L'effort d'abstraction doit donc autoriser des mises en ordre et des comparaisons 52 .

Dans notre étude, la typologie peut s'appuyer sur des variables telles que : l'origine de l'organisation paysanne, sa composition, sa fonction ou son mode de fonctionnement.

L'indicateur lié à l'origine permet de déterminer les forces qui ont suscité l'émergence d'une organisation paysanne. On pourra ainsi rechercher l'origine exogène ou endogène de l'organisation afin d'apprécier son degré d'ancrage par rapport aux groupes sociaux et à l'organisation de la société locale. Cette typologie selon l'origine peut inspirer trois modalités :

- Les O.P de mouvance étatique impliquant les anciennes coopératives et les comités villageois $^{53}$.

- Les O.P de type paraétatique qui sont l'initiative des sociétés de développement (SEMRY, SODECOTOON).

- Enfin, les groupements d'initiative privée qui sont l'œuvre des ONG, des Eglises, des élites locales 54 .

Il faudrait souligner que des zones entières ont été laissées à l'abandon. On a privilégie une approche par filière autour de quelques produits : coton, café, cacao, arachide, ri, ... (voir les O.P, op.cit p. 10-11).

51 Voir J.M. Ela, Quand l'Etat pénètre en brousse : les ripostes paysannes à la crise, Paris, Karthala, 1999.

C'est le cas des type-idéaux, élaborés par Max Weber qui sont des modèles abstraits de la réalité étudiée et dont la cohérence interne s'apprécie par rapport à des schémas opérationnels (le Savant et la politique, trad. Plon 1959. p. 102).

53 L'échec de cette mouvance est dû au fait que les relations entre le centre et la périphérie baignent dans l'ambiguïté : Le pouvoir central continuait à jouir des prérogatives exorbitantes face à une communauté villageoise qu'on voulait pourtant responsabiliser dans l'optique d'un véritable développement autocentré et auto entretenu (J.P Fogui, La tentative d'organisation du monde rural au Cameroun, in Revue sciences et techniques Vol. $4 \mathrm{~N}^{\circ} 1-2$, janvier-juin 1989. p. 82). 
Cette classification a l'avantage de montrer l'influence des pratiques sociales et du fonctionnement des sociétés locales sur les formes d'organisations paysannes et de marquer leur autonomie $^{55}$. Il est évident que les OP de création endogène présentent un maximum de garantie d'indépendance puisque voulues et créées par les paysans eux-mêmes, qui se sont donnés des objectifs conscients et explicites. Sous cette forme, elles pourraient être capables de formuler des revendications conformes aux aspirations du monde paysan et en mesure si nécessaire de mobiliser les masses paysannes dans les actions concrètes.

Seule la combinaison de plusieurs critères peut permettre une approche adaptée de chaque pays.

Ainsi dans un souci opérationnel, DAOUDA Diagne a utilisé un ensemble de critères de différenciation pour cerner la diversité des organisations paysannes togolaises. Il retient entre autres :

- Le mode de création qui peut être endogène ou exogène. Cette dichotomie permet selon l'auteur de déterminer le degré d'autonomie de l'organisation paysanne à l'évidence plus affirmé lorsqu'elle est d'origine endogène.

- L'origine des ressources financières : selon les cas, elles peuvent provenir des cotisations, des crédits ou des subventions.

- La nature des activités (production, collecte...)

- La composition de l'OP (producteurs, investissement collectif) ${ }^{56}$.

La diversité des organisations paysannes entraîne de fait une diversité d'approches permettant d'obtenir maintes possibilités de classification.

Dans le cadre ivoirien, Jean Noël Ferraille et François Rossin retiennent trois variables de classification $^{57}$ :

- une typologie selon le statut des regroupements professionnels qui inclurait les mouvements coopératifs, les associations des producteurs, les syndicats, les comités de développement villageois.

Jean Pierre Prud'homme, Situation et évolution des organisation paysannes et rurales : le Cameroun, Réseau GAO, 1992.

Exemple das migrants de la ville du fleuve Sénégal, Influence de la société Mossi sur le fonctionnement des groupements Naam au Burkina Faso.

56

D. Diagne, Situation et évolution des organisations paysannes et rurales : le Togo, Réseau GAO, 1994, pp 42-50.

57

J.N. Ferraille / F. Rosin, Appui à la structuration de la paysannerie en Côte d'Ivoire : Pour l'émergence de l'organisation professionnelle agricole, Min. de la coopération AFDI 1992, p. 30. 
- une classification selon les domaines d'intervention notamment la fonction économique, l'épargne/crédit, l'organisation du travail, la technique et la vulgarisation, la mutualité, les assurances.

- enfin une classification selon le niveau de professionnalisme ; elle associe les variables telles que le degré d'autonomie dans la prise des décisions, dans la définition des objectifs et dans la planification et le niveau de maturité.

Face au foisonnement anarchique et disparate des OP, on s'aperçoit que les classements et typologies ci-dessus proposés n'ont un sens que s'ils sont utiles à la compréhension de la réalité.

En conséquence, par une approche sociogénétique, il est plausible de proposer une typologie par mouvance qui permette de comprendre les ressorts de l'évolution d'une organisation paysanne. On peut dès lors en distinguer quatre :

- la mouvance administrative, la mouvance communautaire, la mouvance économique, et la mouvance syndicale. Dans la rubrique administrative, on peut regrouper les comités villageois de développement, les organisations des jeunes et toutes les autres organisations dont la création a été impulsée par l'administration et qui exercent des activités à caractère économique et social ; elle se rapproche des "groupes d'intérêts institutionnels ${ }^{58}$.L'on noter que les modes d'intervention dirigiste de l'Etat ont contribué à discréditer auprès des paysans l'idée de la coopérative.

- La mouvance «communautaire » a une vocation plutôt générale et regroupe les organisations enracinées localement et animées par des leaders villageois. Ce sont en général des initiatives locales issues de la rencontre entre le charisme d'un leader et la volonté d'action d'un groupe. Elles intègrent à leurs activités une forte composante sociale (santé, éducation...). Il s'agit selon la typologie d'Almond et de Powell de «groupes d'intérêts associatifs ${ }^{59}$ qui possèdent le degré d'organisation et de spécialisation qui caractérisent les groupes de pressions efficaces, par opposition aux organisations à caractère opportuniste où "groupes d'intérêts non associatif » ${ }^{60}$; la mouvance communautaire est soutenue par une dynamique locale solide.

- La mouvance économique pourrait réunir les organisations paysannes créées à l'initiative des sociétés de développement. Ce sont des sociétés commerciales engagées

Ce sont des organisations formelles crées par l'Etat remplissant d'autres fonctions que l'articulation des intérêts. IV p. 72. ce sont des groupes volontaires et spécialisées dans l'articulation des intérêts.

60

L'expression est toujours de G.A. Almond et G.B Powell. Elle désigne des groupements et non volontaires caractérisés par l'absence de continuité et d'organisation. 
dans la gestion directe d'opérations de commercialisation primaire et d'approvisionnement en intrants. Elles assurent des activités à caractère économique dominant.

- Enfin, la mouvance syndicale : de nature représentative, elle joue un rôle fondamental dans la négociation et la défense des intérêts des bailleurs des fonds ; d'émergence récente, elle regroupe des organisations encore jeunes, où la conscience de faire un métier commun est un fort élément de cohésion ${ }^{61}$. Ces organisations sont surtout présentes dans les filières de culture de rente (café, cacao, coton); elles prennent en général des formes d'action proche du syndicalisme.

Cette émergence quasi-anarchique des OP n'emporte pas toujours aptitude à exercer une véritable pression sur les pouvoirs publics ; seul un regroupement par affinité dans un cadre fédéral peut leur impulser la dynamique nécessaire pour une action syndicale.

\section{La construction macroscopique du pouvoir paysan : des dynamiques fédératives}

La construction microscopique du pouvoir paysan s'est opérée de façon quasi-anarchique. De taille considérablement réduite le O.P se sont avérées incapables de mobiliser les masses paysannes dans des actions concrètes. D'où l'émergence des groupements plus étendus spécialisés dans «l'articulation des intérêts » et possédant le degré d'organisation et de spécialisation qui caractérise les groupes de pressions efficaces ${ }^{62}$. Les formes usitées incluent les unions, les fédérations, les regroupements. Il s'agit des organisations dépassant le cadre villageois et rendues nécessaires par les limites d'une approche exclusivement locale : elles posent comme principe la nécessité d'une approche entre les différents échelles géographiques ${ }^{63}$.

Bien des auteurs dressent un inventaire des facteurs favorables à l'émergence des mouvements paysans. On se trouve en présence d'analyses qui mêlent la référence à un métier (profession agricole), à la défense d'une catégorie sociale (mouvement paysan) ${ }^{64}$. En tout état de cause, les fédérations font partie d'un mouvement paysan en voie de constitution.

Les organisations paysannes et rurales, publication du groupe GAO, Ministère de la coopération, 1995 p. 16.

R.G. Chwartzenberg, Sociologie politique, Paris, Monchrétien, 1988, p. 518.

Les interventions en milieu rural: Principe et approche méthodologiques, Groupe de travail coopération française, Ministère de la coopération et de développement, 1989, pp 54-55.

Voir D. Gentil / M. Ercoirer Situation et évolution des organisations en Afrique noire ? in Revue du tiers-monde, T XXXII, n 128, Oct - Déc. 1994 ; Jacques Berthomé / Jacques Mercoiret, Situation et évolution des organisations paysannes d'Afrique sahélienne: Le Sénégal, Réseau GAO, 1992 ; Jean Claude Deveze, Les Organisations rurales au cœur de la transformation des campagnes africaines. CCCE, Notes rénéotypées Août 1992. 2 p. 
Ce mouvement va au-delà de la somme des organisations, des membres et implique les valeurs, un engagement de type politique (participation à la définition de la politique de développement du pays) et une dynamique plus large que l'activité des fédérations.

L'efficacité de l'action d'une O.P dépend de son autonomie financière et intellectuelle, des objectifs conscients et explicites qu'elles se fixent, de ses rapports avec l'Etat et le reste de la société civile, de sa taille et de son organisation interne. La construction d'un pouvoir paysan fédératif opère selon une double dynamique descendante et ascendante.

- La dynamique descendante ou coopérative est construite par le haut et a une vocation essentiellement économique. Ce sont des groupements de coopératives contrôlés par l'Etat et recevant les appuis des bailleurs de fonds internationaux. Elles ont une mission de commercialisation et d'exploitation des produits. Bien qu'engendrées par le haut, elles peuvent susciter une réelle participation paysanne. C'est par exemple le cas de l'UCOBAM au Burkina Faso. Soulignons qu'elles ne peuvent pas totalement échapper à l'emprise de l'Etat qui cherche toujours à garder de fait le contrôle de l'instance sensée représenter les paysans.

- La dynamique ascendante ou fédérative quant à elle résulte d'unions d'organisations de base soutenues par l'appui d'organismes souvent privés. Ceux-ci constituent des cadres plus souples d'accompagnement facilitant la construction des dynamiques collectives dépassant le cadre local. On peut en discriminer deux modalités : une mouvance plus spécialisée avec des intérêts spécifiques liés à une production dominante. C'est le cas du SYCOV au Mali (Syndicat des producteurs de Coton et Vivriers) issu des Associations villageoises qui ont élaboré une plate-forme de revendications imposant ainsi leur participation aux négociations sur la gestion de la filière coton. Il existe aussi une autre mouvance plus généraliste, aux activités fort variées (Naam au Burkina Faso ou du Conseil des Fédérations Paysannes du Cameroun (CFPC $)^{65}$.

De toute façon, la naissance et le développement d'organisations fédératives correspondent au développement d'enjeux des luttes pour la reconnaissance des mouvements paysans représentatifs. Aussi, tend-on vers la construction d'une société civile africaine ferment d'une force de négociation avec l'Administration et les opérateurs économiques.

Si les interventions de l'Etat se sont faites de façon unilatérale par le truchement d'un développement par projet, il s'est consécutivement développé une politique «manducatoire » avec une propension délibérée d'affecter les financements à des fins de consommaNanga Eboko (centre. BOSAPRAL dans la Lékié. UGCE de la région d'ESSE). Cette fédération vise 4 objectifs fondamentaux : assurer le bien-être des membres, développer la solidarité entre les membres, devenir l'interlocuteur des paysans auprès des pouvoirs publics, défendre les intérêts économiques de ses membres, (voir Jean Pierre Prud'homme, Situation et évolution des organisation paysannes et rurales : le Cameroun, Réseau GAO, 1992). 
tion des gestionnaires et de leurs mandants. Cette conception verticale de la relation centrepériphérie impose la nécessité de l'adoption d'un modèle horizontal ou bijectif qui tienne compte des logiques et des rationalités propres au monde rural, contraintes qui structurent largement les possibilités d'intervention de l'Etat. Cela inspire une critique plus large d'Edgar Morin sur le problème de l'imposition des modèles : «La prise de conscience de la grande carence des modèles est le préliminaire à tout progrès politique et social dans l'idée de développement ${ }^{66}$.

\section{Les contraintes périphériques et la modulation des interventions de l'etat en milieu rural : la co-production de l'ordre social}

La mise en cause du modèle tutélaire de l'action publique et la prise en compte subséquente de la dimension anthropologique de l'intervention publique ne s'ouvre pas sur l'individualisme méthodologique; il s'agit plutôt d'une co-production de l'ordre social. Dans cette perspective bien plus qu'ailleurs, le tout est plus que la somme des parties. L'arrimage de la politique économique à celle de l'intégration nationale, a produit un effet d'occultation et même de négation de la pluralité des rationalités périphériques à travers l'hypertrophie du pouvoir central ${ }^{67}$. C'était le temps de l'arrogance hégémonique de rhizome providence et du conformisme institutionnel. Or, en considérant les logiques et les stratégies propres aux acteurs locaux, on pourrait imaginer un modèle moins abstrait qui exprimerait mieux les influences réciproques entre le centre et la périphérie. Il s'agit d'un «modèle co-productionnel» ${ }^{68}$ de l'action publique appréhendé comme un processus transactionnel.

De même, considérer les O.P comme des «acteurs en réseau » ${ }^{69}$, c'est révéler la part du travail de «subversion de territorialité ${ }^{70}$ qu'elles réalisent. Elles ne sont donc pas des acteurs désincarnés. Elles se trouvent impliquées dans une structure de jeu interactionniste qui n'inclut plus seulement l'Etat, mais également les appareils de développement, les

J. Champaud, «Pouvoir central, pouvoirs locaux et développement au Cameroun» in Etats, Pouvoir et espace dans le tiers-monde, sous la Direction de C. Bataillon, Paris, PUF, p. 153-165.

J. Onana, «Changements démocratiques et économie du régime de gouvernance publique en Afrique. Pour une coproduction de l'action publique ». in Gouvernance partagée : la lutte contre la pauvreté et les exclusions, $2^{\mathrm{e}}$ conférence internationale régionale sur les sciences administratives, providence University press, Yaoundé 14-18 juillet 2003 p. 190-191.

69

M.C. Smouths, Les Nouvelles relations internationales, op.cit. p. 23-219; voir aussi une bonne définition, L. Sfez, «Le réseau du concept initial aux technologies de l'esprit contemporain », cahiers internationaux de Sociologie, janvier-juin, 1999, p. 5-27.

70

F. Constantin, «L'Informel international ou la subversion de la territorialité » in B. Badie et $S$. Marie Claude (dir.), L'Internationale sans frontière, Paris, l'Harmattan. Cultures et conflits, 1996, p. 311-345. 
bailleurs de fonds, les organisations non gouvernementales d'aide au développement, les opérateurs économiques etc.

Afin d'indiquer le sens d'une intervention étatique qui n'occulte plus les acteurs périphériques, il convient de relever les contraintes propres au paysannat.

\section{A. L'hétérogénéité des situations et des rationalités paysannes}

Les organisations paysannes devraient être envisagées dans le langage propre de leurs cultures réceptrices. Les catégories culturelles instruisent avantageusement sur la plasticité des catégories fondatrices de l'action publique ${ }^{71}$. Le monde rural présente fondamentalement des situations très diverses du point de vue structure, technique et économique. De ces diversités découlent des logiques sociales tout aussi divergentes. Le fait est que le démembrement de l'intervention publique est inévitable ${ }^{72}$; l'exercice empirique consisterait en une identification des situations rurales à l'évidence fort diverses-, des groupes cibles et catégories intervenantes, et en leur articulation entre les différentes échelles géotemporelles.

\section{La diversité des situations en milieu rural}

L'Etat bureaucratique à travers le développement par projet, les avait conçues de façon uniformisante, en accordant une attention plutôt distraite à leur très grande diversité. C'était en quelque sorte «une intervention aéroportée d'implant d'une modernité inadaptée » ${ }^{73}$ et enfermée dans l'ordre bureaucratique.

Or, le monde paysan présente une hétérogénéité structure d'abord perceptible au niveau des grands clivages sahel/zone forestière. Cette infinie diversité s'observe même dans les espaces plus limités. Au Burkina Faso par exemple, une stratification du monde rural en six zones appelant des réponses différenciées sur le plan technique a été effectuée dans le Yatenga sur la base de trois critères (pluviométrie, nature des sols et densité de la population). Pour cerner le caractère diversifié du monde paysan, il semble judicieux de procéder à l'identification des composantes de production et du niveau de maîtrise qu'en ont les producteurs ; d'où la nécessité d'une description du milieu physique (nature et état des sols,

J.F. Baré, «L'évolution de l'intervention publique comme exercice d'anthropologie historique ». in J.F Baré (dir.), Evaluation des politiques de développement, Paris, l'Harmattan, 2001 p. 98.

72 Baré, op.cit P. 133.

73

Ndembou, « Le développement rural par projet ... », op.cit., p. 294. 
climat etc.) et d'une détermination du mode d'occupation de l'espace (densité de la population, stratification de l'espace); mais aussi les caractéristiques de la mise en valeur du milieu.

Les leçons de l'expérience malienne confirment le caractère malléable et ambivalent des structures et des actions paysannes ${ }^{74}$ : d'un côté, elles subissent les pressions de l'environnement social et tendent à reproduire les systèmes en place; de l'autre, elles sont susceptibles d'introduire les modifications légères mais perceptibles grâce au « développement des marges de manœuvre et à l'élargissement du champ des possibles ${ }^{75}$.

Aux situations agro-écologiques différentes, s'ajoutent des situations économiques éminemment variées.

Les pesanteurs économiques constituent un critère pertinent de la politique d'intervention en milieu rural. En effet, il est des zones rurales où l'exploitation d'une filière (café, coton, cacao) peut procurer des avantages comparatifs aux paysans. De même, la politique d'intervention de l'Etat doit au préalable tenir compte des distinctions entre zones suivant qu'il existe ou non une rente de situation (existence d'un marché, d'une industrie, d'une zone frontalière), des niveaux de développement des infrastructures (réseaux de communication, organisation des transports).

Dans les espaces agraires réduits, les indicateurs tels que la superficie des exploitations, le capital, la stratification sociale, la main d'œuvre peuvent aider à élaborer des typologies qui traduisent cette grande diversité des situations économiques. Il n'est donc pas stratégique pour l'Etat de couler le monde rural dans un moule uniformisant, qui ferait abstraction des contingences agro-écologiques qui structurent plus ou moins favorablement l'ordonnancement du milieu rural.

Cette préoccupation est bien large et implique les formes de logiques sociales.

\section{La diversité des logiques endogènes}

Les logiques endogènes du monde rural sont inappréhendables au premier degré. Ce sont des logiques cachées propres à un système et qui lui permettent d'assurer son autorégulation existentielle. Ce sont ces considérations réalistes qui peuvent aider à refouler les pré- 
jugés tenaces de la thèse de la «greffe de l'Etat» ${ }^{76}$, car il existe une logique d' «indigénisation de l'action hégémonique de l'Etat» ${ }^{77}$. Autrement dit, il faudrait lire la réalité dans la structure mentale des acteurs de la paysannerie.

Dès lors qu'une telle exigence est satisfaite, il devient relativement aisé d'élaborer quelques indicateurs permettant d'identifier et de caractériser ces logiques. Les travaux de Marie Rose Mercoiret et de Jean Claude Devèze nous aident à répondre à certaines préoccupations locales des paysans ${ }^{78}$. Il s'agit pour ces auteurs de battre en brèche l'indigence d'une analyse qui confinerait les logiques paysannes à l'archaïsme et au primitivisme. La problématique tournerait alors autour des objectifs économiques et des finalités sociales qui déterminent les comportements supposés irrationnels des paysans ${ }^{79}$.

Ceux-ci varient selon les contextes : l'environnement socio-économique peut être plus ou moins sécurisé, les écosystèmes peuvent s'avérer fortement dégradés, les terres encore neuves; ou selon les caractéristiques structurelles des différentes exploitations (la capacité objective à prendre des risques, le statut social des paysans etc.).

Ces facteurs influencent doublement les stratégies paysannes ; ainsi, dans les zones fortement fragilisées, la logique de survie est dominante, le choix opéré étant à cour terme avec une limitation maximale des risques. Par contre, les logiques d'accumulation caractérisent les zones plus favorables et certaines catégories sociales.

Les modalités d'intervention en milieu rural doivent prendre en compte non seulement la logique sociale endogène, mais également les structures paysannes existantes. La situation de ce point de vue est très variable d'une région à l'autre.

\section{La diversité organisationnelle du monde rural : le poids des rationalités structurelles}

Une reformulation des stratégies d'intervention de l'Etat en milieu rural suppose aussi une prise en compte de la variété des types d'organisations paysannes existantes ${ }^{80}$. En effet,

B. Badie / P. Birnbaum, Sociologie de l'Etat, Paris, Grasset, 1979, p. 180-181.

77

78 Structure, Rhizome ailleurs ... op.cit., p.29.

Notamment, les interventions en milieu rural : Principes et approche méthodologique, groupe de travail coopération française 1989.

79 Il est d'ailleurs superflu de remuer la fameuse thèse faussement épistémologique de l'évolutionnisme unilinéaire (voir L.H. Morgan, Ancient society, 1877).

80

Cette exigence s'observe au Burkina Faso où les groupements villageois sont des partenaires privilégiés. 
l'origine de la structure, sa nature, son effectif et son impact qualitatif, la gestion du pouvoir au sein de l'organisation (récupération de la structure par les notables, émergence des nouveaux leaders, inamovibilité des responsables, fonctionnement démocratique) constituent des indicateurs qui, mis à la dispositions de l'Etat, des bailleurs de fonds et des organisations d'aide au développement peuvent aider à une articulation des modalités d'intervention. Toutes ces variables structurent de façon déterminante le rendement des organisations paysannes. Un tel phénomène est observable au Burkina Faso où les groupements villageois sont des partenaires habiletés. Cette aptitude est bien plus perceptible lorsque les organisations paysannes sont confirmées, qu'elles soient le résultat d'interventions antérieures ou en cours (Mali du Sud) ou d'initiatives endogènes relayées par les ONG (Associations villageoises de développement); cette action est moins aisée quand coexistent des structures paysannes résultant d'actions administratives (communautés rurales), politiques (comités), économiques (coopératives étatiques, culturelles), imposées ou spontanées, crédibles ou discréditées, articulées ou non sur un héritage traditionnel plus ou moins vivace.

L'analyse de la diversité de situation et des rationalités paysannes permet ainsi d'effectuer des choix d'intervention qui suscitent nombre de questionnements pour l'essentiel résumés ci-dessous :

Faut-il privilégier les zones les plus porteuses du point de vue économique au détriment des zones marginales ? Mise-t-on sur les paysans les plus dynamiques ou sur la grande masse des paysans? Doit-on doter les organisations paysannes d'un mécanisme de stabilisation des prix des produits ou d'un appareil de vulgarisation ? Le rapport coût/bénéfice doit-il être envisagé dans une perspective à court ou à long terme ? Peut-on parler de rentabilité lorsqu'on préserve l'environnement? Doit-on s'attaquer aux problèmes fonciers dans certaines régions?

Seule la prise en compte de la diversité des situations et des rationalités peut faciliter la négociation entre les intérêts différents en créant une conjonction sociale favorable à l'épanouissement du monde rural. Mais, on s'aperçoit que la dynamique des organisations paysannes et les stratégies d'intervention de l'Etat et des bailleurs de fonds n'intègrent généralement pas les différentes échelles géographiques et temporelles. Cet empirisme est générateur d'incohérences qui requièrent une mise en ordre logique

\section{B. L'articulation entre les différentes échelles géotemporelles}

Sur le plan géographique, il y a un ordonnancement territorial impliquant le local, le régional et le national. Les échelles de temps ont trait au moyen, long ou court terme. Ces deux dimensions sont déterminantes dans la construction du pouvoir paysan. 
L'articulation de l'échelon local avec les autres échelles géographiques est rendue nécessaire par les limites d'une approche exclusivement locale. IBN KHALDUN le soulignait déjà au XIV ${ }^{\text {ème }}$ siècle que : «les besoins d'une collectivité ne peuvent résolus que par la coopération ${ }^{81}$.

\section{La conjonction entre les différentes échelles géographiques}

Une dynamique locale autarcique qui conduirait les OP à n'exploiter que des petits espaces agraires, réduit les possibilités internes d'échanges et ne permet pas la couverture directe de tous les besoins. Cette conception désincarnée des OP engendre une logique contre-productive. En langage systématique, elles seraient incapables d'exercer la fonction de régulation des exigences ${ }^{82}$. En effet, les problèmes posés par l'approvisionnement, la commercialisation, les formations techniques spécialisées ne peuvent à priori recevoir une réponse locale ; de même, les interactions villageoises et leurs articulations avec le secteur moderne ne peuvent être appréhendées à partir du seul niveau local. L'autarcie conduirait à une surcharge qualitative tenant à la complexité des exigences adressées au système et portant sur les problèmes délicats, difficiles et longs à traiter.

Une première formule d'articulation consisterait à assurer la conjonction entre le niveau local et l'échelon régional. Ce pôle devra se constituer en point d'appui aux initiatives locales dans les domaines de l'approvisionnement et de la commercialisation (organisation des échanges, voies de communication), de la formation professionnelle ${ }^{83}$, du financement (crédit). Bien plus, le cadre régional se posera en lieu de confrontation, d'échange d'expérience et d'arbitrage des logiques locales. Ce faisant, les associations régionales joueraient un rôle intégrateur en assurant une cohérence bénéfique «par le haut »; il est question des régions agro-écologiques et économiques (et non des régions administratives) aptes à impulser un véritable développement rural qui ne soit pas exclusivement agricole mais largement diversifié.

Cette régionalisation du monde paysan doit bien entendu résulter d'une négociation entre les différents secteurs économiques, et supposer une véritable politique nationale. 
Ce deuxième palier de l'articulation est éminemment important même s'il suscite des interrogations sur le rôle de l'Etat dans un processus de libéralisation économique ${ }^{84}$. La définition d'une politique agricole nationale ne constitue pas une nouvelle «capture de la paysannerie» par l'Etat ${ }^{85}$. Les contraintes de l'économie libérale et des dynamiques locales lui imposent une politique adaptée.

\section{L'articulation des interventions de l'Etat avec les différentes échelles de temps}

Le développement obéit aussi à des inscriptions temporelles. Le temps est un élément fondamental de la gestion des projets de moyen terme. La propension était d'opter pour une visée temporelle à moyen terme. Ce choix était guidé par la politique des bailleurs des fonds qui avaient pour souci d'éviter de créer des situations de rente et surtout de ménager des possibilités de retrait encas de disfonctionnement. Pourtant le moyen terme constitue sans doute l'échelle de temps la moins favorable pour concevoir une approche de développement sectoriel aux conséquences immédiates et répondant à des urgences; le moyen terme demeure relativement court pour que se mette en place des processus de développement durables avec des orientations à long terme.

Face à ce dilemme, une judicieuse articulation temporelle consisterait précisément à mobiliser les moyens de se libérer de cette contrainte pour définir des perspectives à long terme d'où se déduiraient des stratégies à moyen terme. La visée temporelle à court terme n'est toute fois pas abandonnée ; car les programmes d'urgence sont susceptibles de lever les contraintes les plus immédiates qui grèvent le temps productif (collecte du bois, puisage de l'eau pour les femmes du Sahel etc.) et qui parfois aggravent l'instabilité paysanne (exode saisonnier).

On peut ainsi imaginer des microprojets exigeant des moyens financiers modestes, pouvant rapidement être discutés avec les paysans et mis en place urgemment (maraîchage, embouche des petits ruminants, banques des céréales, moyens de transport, moulins etc.). Ces réalisations de petites dimensions crédibilisent l'intervention de l'Etat et des bailleurs des fonds dans le monde rural. Elles permettent également de dégager du temps pour une réflexion approfondie avec les villageois afin de fixer les orientations à long terme ; c'est

Rhizome ne doit pas se désengager du processus de development rural, il doit avoir une politique qui le favorise en matière de prix, de credit, du foncier, il ne faudrait pas qu'il tombe d'un excès à l'autre.

G. Hyden, La crise africaine et la paysannerie non-capturée, Politique Africaine, $\mathrm{N}^{\circ} 18$, année 1985, p 93-113. 
un formidable moyen d'apprentissage par les paysans, des objectifs, des règles et des mécanismes d'organisation.

Somme toute, il s'agit de prendre en compte les «temps multiples » permettant l'adaptation des objectifs, des modes d'organisation, des méthodes et des moyens financiers à la durée.

\section{Conclusion}

Les OP se sont révélées comme des acteurs rebelles à la formule bureaucratique classique qui n'a envisagé le développement en milieu rural qu'à travers des projets sans prise effective sur les réalités périphériques. Les OP participent ainsi à la construction d'une société civile en gestation. Ce sont des véritables « groupes de pression » qui modulent désormais une échancrure dans le corps hermétique de l'Etat. Le vocabulaire de la régulation sociale a subi une mutation paradigmatique: l'on passe insensiblement d'un modèle tutélaire de gestion à un modèle de co-production de l'action publique avertie de l'impérieuse nécessité d'une gestion participative des politiques publiques.

Bien des auteurs imaginent la possibilité d'une inscription de l'action internationale pour le développement dans les imaginaires sociaux $^{86}$; il y a certes un fort soupçon de dévelopmentalisme qui biaise une telle proposition; mais si nous l'envisageons à travers le paradigme de la co-production, l'illusion de l'évolutionnisme s'estompe d'elle-même. De la sorte, l'on tournerait en avantage «les formidables recompositions sociales et idéologiques dont sont porteuses les sociétés civiles, au lieu que celles-ci soient tenues pour des purs fantasmes structurels» ${ }^{87}$.

A. Faure et al., La construction du sens dans les politiques publiques. Débat autour de la notion de référentiel, Paris, L'Harmattan, 1995. C'est aussi la perspective tracée par C.H. Giddens, The constitution of the society, policy press, Cambridge 1984. 